\section{A FORTRAN IV program for a Bayesian analysis of a posteriori comparisons in the completely random design}

\author{
JAMES E, POWERS \\ State University of New York at Albany \\ Albany, New York 12222
}

This program derives posterior distributions on a posteriori contrasts among treatment means in the completely random analysis of variance. To employ the program, the user must specify prior probabilities on a set of orthogonal linear functions of the treatment means as described by Powers (1970). In so doing, he has implicitly placed prior distributions on all possible linear contrasts of those means and the posterior distributions for these contrasts are obtainable (Powers, 1971). The program may be used any time the population variance is known. It is also appropriate in most cases when the sample size in each treatment is greater than four even if the population variance is known (Hughes \& Powers, 1971).

Input. The input of the program consists of the coefficients of, and the prior probabilities for, the original set of orthogonal linear functions of the treatment means, the coefficients of the set of a posteriori contrasts to be analyzed and either raw scores or summary statistics in the form of treatment means.

Output. The program first prints out the treatment means, the estimate of the population variance and the sample size in each treatment. For each function in the original set of orthogonal linear functions, output consists of the coefficients, the values for which prior probabilities were specified, the prior, likelihood and posterior distributions, and the posterior mean and standard deviation. For each treatment mean and each of the set of a posteriori contrasts the coefficients are printed. Then the calculated values for the means and contrasts with their associated posterior probabilities are listed in the form of frequency distributions.

Restrictions. The program may be employed for a completely random analysis of variance having from two to eight treatments and equal sample sizes in the treatments. Prior probabilities may be specifjed for up to 30 values of each of the orthogonal set and posterior distributions may be obtained for up to 30 a posteriori contrasts at a time. Most of these limits may be increased by simple changes in dimension statements. Storage requirements for the program are not excessively large and should not exceed core on most machines. The program was developed and checked on the UNIVAC-1108 system at the State University of New York at Albany.

Availability. A listing and writeup of the program are available free of charge from James E. Powers, Education 232, State University of New York at Albany, 1400 Washington Avenue, Albany, New York 12222.

\section{REFERENCES}

Hughes, F., \& Powers, J. On replacing the population variance by its sample estimate in a Bayesian analysis of planned orthogonal comparisons. Experimental Publication System of the American Psychological Association, 1971, 10, Ms. No. 352-24.

Powers, J. A Bayesian approach to the completely random design. Paper presented at the meeting of the Northeastern Educational Research Association, Grossinger, New York, November 1970.

Powers, J. A Bayesian procedure for a posteriori comparisons in the completely random design. Paper presented at the meeting of the Northeastern Educational Research Association, Grossinger, New York, November 1971.

\section{Standard error of weighted means in a two-stage sampling model with unequal first-stage units}

\author{
B. BRONFMAN* \\ Oregon Research Institute, Eugene, Oregon 97403
}

Since the costs of executing a true random-sample survey have risen sharply over the past few years, some alternative plans are increasingly coming into use. One reasonably cost-effective alternative is the two-stage sampling technique. A random sample of census tracts, or first-stage units, is first made, and a random selection of respondents within selected census tracts, or second-stage units, is then made. This program calculates weighted means and standard errors of means for the two-stage sampling model, with unequal first-stage units, for any set of consecutive data sets. Standard errors of means are calculated after a procedure suggested by Sukhatme and Sukhatme (1970), as shown in Formula 1:

$$
\hat{S}_{1}\left(\bar{y}_{s_{2}}^{\prime \prime}\right)=\sqrt{\left(\frac{1}{n}-\frac{1}{N}\right) \frac{1}{n-1} \sum^{n} u_{i}^{2}\left(\bar{y}_{i\left(m_{i}\right)}-\bar{y}_{s_{2}}^{\prime \prime}\right)^{2}+\frac{1}{n N} \sum^{n} u_{i}^{2}\left(\frac{1}{m_{i}}-\frac{1}{M_{i}}\right) s_{i y}{ }^{2}}
$$

